\title{
METAPHORICAL INTEGRATIONS IN KURDISH RIDDLES
}

\author{
Rahman Veisi Hasar \\ Department of English Language and Linguistics \\ University of Kurdistan, Sanandaj, Iran \\ e-mail:veisirahman@yahoo.com \\ Ebrahim Badakhshan \\ Department of English Language and Linguistics \\ University of Kurdistan, Sanandaj, Iran \\ e-mail:badakhshane@gmail.com
}

\begin{abstract}
This paper aims at investigating the metaphorical integrations of riddles in the Kurdish language. For this purpose, 100 Kurdish riddles are analyzed according to the blending theory of metaphor (BT). Accordingly, the riddles are classified into three groups. The first one, which we call a contingent metaphor, involves those riddles shaped by metaphorical mapping between the precedent and the sequent as input spaces, which are then combined and compressed into the blend space. The linguistic structure of a contingent riddle includes the precedent space and some of the conceptual correspondences without any explicit reference to the sequent space. The possible candidates of the hidden sequent space are conditioned by the image-conceptual structure of the precedent space. The second group, which we call a discordant metaphor, includes those riddles that are based on a complicated metaphorical integration between the precedent and the sequent as input spaces: elements and relations of input spaces are cross-mapped, and then they are mixed and compressed into the blend space; and ultimately, a kind of disanalogy between spaces is brought about by backward projection. The linguistic structure of a discordant riddle includes two contradicting parts. The first part indicates the metaphorical integration, and the second shows the disanalogy. The candidacy of a possible sequent space is conditioned by the structure of the precedent space and by the differences highlighted by the backward projection. The metaphorical riddle in both groups is a specific kind of deliberate metaphor which invites the riddlee (addressee) to view (guess) the sequent in terms of the precedent. The deliberateness of the metaphorical riddle leads the riddlee to construct numerous metaphorical mappings between the precedent space and many possible candidates for the sequent space. The third group includes the non-metaphorical riddles not applying any kind of metaphor.
\end{abstract}

Keywords: blending theory of metaphor, contingent metaphor, discordant metaphor, Kurdish language, metaphor, riddle 


\section{INTRODUCTION}

The riddle is a very important and indispensable part of the Kurdish folklore in a sense that its cultural role might be traced back to the ancient mythologies in the Old Iranian era. According to one of the Iranian mythologies, a hero sacrifices one hundred horses, one thousand cows and ten thousand sheep for a goddess to help him to find the answers of some riddles (Khaleqi Mutlaq 2002: 169). The ancient idea of the riddle has been verbalized by many synonymous

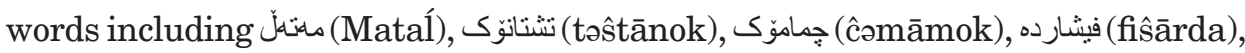
or شَتَّكاني (ŝatēkānē) in the Kurdish language. Among other words, the word təŝtānok may describe the very nature of the riddle more vividly; it literally means what-is-ness. In fact, it refers to the enigmatic nature of the riddle, and demonstrates the question-oriented discourse of the riddle. The riddle in the Kurdish culture contributes to many different social functions. The researchers (see Zolfaqhari 2013: 108-110; Ahmadi 2010: 14) have named many functions of the riddle in the Iranian culture including education, improving the intelligence of the children, entertainment, competition among people, and propagating the ideological and religious ideas.

The ethnic context in which riddles are mainly exercised is called ŝaw nəŝini in the Kurdish language; it literally means sitting around each other in the night. The Kurdish culture was mainly based on an agricultural society, and people were busy throughout the day. But they could use their spare time at night to tell stories (Bayt) or ask riddles. ${ }^{1}$ Elderly people were mainly narrators and riddlers, and younger ones were narratees and riddlees. With regard to riddles, elderly people as the holders of the ethnic wisdom ask riddles from younger ones to evaluate their familiarity with the ethnic heritage. The riddlers often promise a gift or a prize to those who can find the correct answer. In all areas of Kurdistan, the riddle is a common tradition. The Kurdish riddles mainly cover six general topics including information about physical objects, animals, plants, instruments, human beings, and religious-scientific knowledge (see Shohani 2014; Ahmadi 2010: 19-23). The Kurdish riddles, similar to riddles in other cultures, mainly have a kind of two-part structure: the question along with some clues and the hidden answer. In the following example, the riddle involves the question (or the question image) and the answer.

1. Yak pay hasu yak ĉāw.

It has one leg and one eye. (needle)

The riddle as such is combined of two parts, namely the precedent (the question or the question image) and the sequent (the answer of the riddle). The former includes some properties, images, propositions, or puns providing some clues 
for surmising the latter (see Harries 1971: 388; 1976; Dienhart 1999: 101-102). But, the relationship between the precedent and the sequent varies according to the figurative devices applied in the riddles. For example, in non-metaphorical riddles such as conundrums or puns it is the phonetic similarity (not a conceptual relationship) linking the precedent and the sequent (see Dienhart 1999). Adding to this, in most of the non-metaphorical riddles, the phonetic, syntactic, or semantic ambiguities are the "block elements" of the riddle, which should be removed to find the right answer. The removal of the block elements is done by referring to linguistic codes and grammatical limitations of a given language (see Green \& Pepicello 1984: 189). In other words, the riddlee tries to find the sequent by disambiguating the precedent. Let us have a look at the following examples:

2. What turns but does not move? Milk (from Dienhart 1999: 107).

3. Why is coffee like the soil? It is ground (ibid.).

In riddle 2 , the riddle is made by an ambiguous phonetic form (turn) having two different meanings (rotating and changing). The riddlee should disambiguate the word by choosing the second meaning (changing), which is compatible with the riddle. Also, in riddle 3 the ambiguity emerges as a result of the homophony between the noun lexeme of ground /grawnd/ and the past participle of the grind /graynd/ as the intended meaning in the riddle. Accordingly, the linguistic structure and the superficial elements in non-metaphorical riddles are especially important for analyzing the riddle. By removing the ambiguities, comparing the phonetic forms, or analyzing the grammatical errors, the riddle can be solved.

However, there are some riddles that may not be relegated to a kind of superficial linguistic ambiguity, simply because they are based on an underlying metaphor. Metaphorical riddles are based upon a cognitive comparison between the precedent and the sequent, consequently they may not be solved by eliminating the superficial ambiguities or grammatical errors. First the underlying comparison between the two parts is made, and next the linguistic structure is presented (see Green \& Pepicello 1984: 201). Köngäs-Maranda (1971) believes that there is an underlying metaphorical comparison between the elements of the precedent and the sequent prior to the genesis of the linguistic structure of the metaphorical riddle. Then, the given term as the precedent (the core of the riddle image) is compared to the hidden term as the sequent (the answer) according to some premises (the clues). The following table is presented by Köngäs-Maranda (ibid.: 17) to explain the metaphorical riddle of one pig two snouts (plough). 


\begin{tabular}{|l|l|l|l|l|}
\hline Terms & & Premise & Premise & \\
\hline & & constant & variable & \\
\hline Given & A pig & has snouts & two & image \\
\hline Hidden & A plough & & one & answer \\
\hline
\end{tabular}

As is evident in the above table, the metaphorical riddle is made by imageconceptual comparison, and may not be reduced to superficial linguistic features. The conceptual aspects of the metaphorical riddles should be explained from a cognitive perspective. For this purpose, let us review the concept of metaphor and its relevance to the riddle in cognitive semantics.

Metaphor as a cognitive omnipresent phenomenon satisfies many heterogeneous functions. Its functions range over a variety of cognitive abilities, from understanding and feeling (see Lakoff \& Johnson 2003 [1980]; Lakoff 2007; Kovecses 2004 [2000]) to conceptualizing the socio-cultural and theological experiences (see Kovecses 2005; Musolff 2004; Gomola 2010; El-Sharif 2012). Besides all these extremely important functions, metaphor can play an important role in a different cognitive ability, namely guessing. If the metaphor is utilized for understanding and experiencing one concept (target domain) in terms of another (source domain) (Lakoff \& Johnson 2003 [1980]: 5), it may also be used for guessing one kind of thing in terms of another. The metaphorical guessing is realized in the metaphorical riddles. In this regard, the riddle is characterized as a metaphorical cognitive phenomenon beyond its linguistic manifestations. Therefore, the given precedent and the hidden sequent are interpreted as the source and target domains respectively, and the premises are read as the linguistic manifestations of the conceptual correspondences between the domains. Then, the metaphorical riddles are based on the conceptual mapping between the precedent as the source domain and the sequent as the target domain. For example, the metaphorical riddle Which is the animal that has four feet in the morning, two at midday, and three in the evening? is based on conceptual mappings among precedents including day and journey and sequents involving life of a human and human life respectively (see Kovecses 2010 [2002]: 11). Consequently, this riddle is based on conceptual metaphors of "THE LIFE OF HUMAN BEINGS IS A DAY" and "HUMAN LIFE IS A JOURNEY". The linguistic manifestation of the riddle includes the source domain (precedent) and some of the conceptual correspondences without any explicit reference to the sequent. With regard to the hiddenness of the sequent part, the metaphorical correspondences between the domains are accomplished by the riddlee's guessing. In other words, it is the riddlee who makes various metaphorical correspondences between the source domain (precedent) and the 
target domains (the possible candidates for the sequent) according to his or her guesses. Composed of explicit image-concepts and a hidden answer, the metaphorical riddle invites the riddlee to guess the latter in terms of the former. In other words, the riddler presents a kind of deliberate metaphor (see Steen 2011: 84) whereby he or she asks the riddlee to view and guess (consciously) the latter (target domain) in terms of the former (source domain) on the basis of a metaphorical comparison.

The blending theory (see Fauconnier \& Turner 2002) as a cognitive theory can explain the complicated cognitive process underlying the metaphorical riddles in more detail. Let us illustrate this theory via explaining the metaphorical integration of the ECONOMIC COMPETITION IS A BOXING MATCH as a conceptual metaphor. According to this theory, every metaphor is composed of four conceptual spaces including two input spaces, the generic space, and the blend one. Accordingly, the input spaces consist of the space of economic competition and that of a boxing match. Also, there is a generic space which involves the shared elements of the input spaces (two opponents, the same goal, and the competition). It is the generic space that makes the metaphorical integration possible; in other words, no integration between the input spaces would be accessible without some shared abstract properties. Finally, there is a blend space in which the elements of the input spaces are combined and compressed; the final conceptual fusion brings about the conceptual metaphor. Therefore, the metaphorical riddle may be seen as a kind of conceptual integration; the precedent and the sequent as input spaces are cross-mapped, and consequently they are combined and compressed into the blend space to give birth to a fusional metaphorical construction (see Fauconnier \& Turner 2002: 119, 49; Rapone 2012). Accordingly, a metaphorical riddle involves four spaces: the precedent and the sequent as input spaces, the blend space (the metaphorical fusion of the inputs), and the generic space (shared elements of the input spaces). As Rapone (2012) puts it, one of the input spaces as the sequent is implicit, but the precedent as the explicit input space, the blend space, and the generic one may supply some clues for the riddlee to guess the hidden sequent space. By relying on information from these three spaces, the riddlee can guess the right answer.

Owing to the lexical and structural ambiguities of the riddle, they may have more than one answer. The procedure of guessing the sequent for both the metaphorical and non-metaphorical riddles is determined by potentiality and indeterminacy. In other words, the clues are not always sufficient for pointing out the specific sequent existing in the mind of the riddler. Accordingly, there may be more than one possible candidate (for the sequent) that can satisfy the clues presented by the precedent. It is because of this that determining the right sequent is always dependent on the will of the riddler (see Ben-Amos 1976: 249). For example, let us look at a non-metaphorical Kurdish riddle. 
4. Helka daka, mriŝk nya.

It lays eggs, but it is not a bird. (turtle)

The answer to this riddle can be turtle, lizard, or even fish. It is the riddler who determines which one is the correct sequent. The potentiality of the sequent refers back to the insufficiency of the clues in the precedent. It should be noted that in some occasions, the riddler intentionally uses ambiguous and vague words in order to deceive the riddlee (see Weiner \& Palma 1993: 189).

The riddle is always based on a reciprocal interaction between potentiality and cognitive operation of guessing. Therefore, any research on the riddle should take the dialectics of cognition and potentiality into consideration. In other words, it should illustrate the potential metaphorical integrations presented by the riddlee's guesses motivated by the ambiguous and indeterminate structure of the riddle. In this regard, the present paper aims at scrutinizing the metaphorical structure of the riddles in the Kurdish language by applying the blending theory of metaphor. It tries to show the triangular dialectics of the metaphor, guessing, and potentiality in Kurdish metaphorical riddles. The riddles under consideration are common in Kurdistan of Iran. They are used by people in different cities like Sanandaj, Boukan, and Saggez.

In addition to the introduction, the present article is comprised of four chapters. The first one spells out the methodology of the paper in detail, the next deals with the typology of the riddles in the Kurdish language. In this chapter, the conceptual structures of the riddles are explained according to the blending theory. The third chapter presents some implications for the process of metaphorical guessing, and finally, the conclusion sums up the results and implications of the paper.

\section{METHOD AND MATERIALS}

The data of the present study are based on a Kurdish dictionary of riddles (Sarseyfi 2010). The book involves 600 riddles along with their answers. Each entry involves a riddle and its answer. Among all the entries, 100 riddles were selected randomly from the alphabetical list of the dictionary. The linguistic structure of each riddle involves the precedent and the clues, and lacks any kind of explicit reference to the sequent (answer). By investigating the relationship between the riddles as the precedent and their answers as the sequent, this paper tries to elucidate their possible metaphorical relationships. The paper attempts to shed light on the ways the precedents refer metaphorically to the hidden sequents in the riddles. 
To recognize the metaphorical usage of the words in the riddles, the method of metaphor identification procedure (MIP) (see Pragglejaz Group 2007) was applied. According to this method, if the contextual meaning of a word contrasts its basic meaning, and also if the former is understood in comparison to the latter, the word is marked as metaphorical. How can this method be applied to riddles? To illustrate this procedure, let us consider two entries of the book (Sarseyfi 2010).

\section{Qalây gači ret nya bəči.}

A plaster castle, you cannot enter it. (egg)

6. Ž̉n nya pyâwiŝ nya, be dâyku bâbiŝ nya.

It is not a woman (a married one) and not a man (a married man), it has father and mother. (a single girl)

Considering riddle 5, the precedent includes the phrase plaster castle. The basic meaning of this phrase refers to a kind of building. But the precedent of plaster castle refers figuratively to a white egg (the sequent) in this riddle. Thus, it can be argued that the contextual meaning of the plaster castle in this riddle is white egg. Besides, the contextual meaning (white egg) is in contrast to the basic meaning (a kind of building); furthermore, the former is understood in comparison to the latter. Regarding MIP, it can be concluded that the plaster castle is used metaphorically in this riddle. Consequently, we regard the literal meaning of the precedent as its basic meaning, and consider the sequent as its contextual meaning in the metaphorical riddles.

But in riddle 6 , the words in the precedent part are not used metaphorically. The precedents including woman, man, father, and mother, and the sequent involving a single girl are not understood in comparison to each other. However, they are related to one another in terms of some distinctive features such as male, female, married, and single. Besides, the sequent may not be the contextual meaning of the precedents, but they are separate meanings that are related to each other in terms of some superficial linguistic ambiguities. Therefore, the precedent of non-metaphorical riddles does not satisfy the conditions of MIP.

To analyze the conceptual integrations of the metaphorical riddles, the blending theory of metaphor (Fauconnier \& Turner 2002) was applied. In this regard, the entry as the precedent and the answer as the sequent were considered as the input spaces. The metaphorical output brought about by fusing the input spaces was considered as the blend space. The common properties of the input spaces were regarded as the generic space. The linguistic manifestation of the riddle contains the input space of the precedent, and also some elements of the blend space, but it lacks any kind of direct reference to the hidden sequent space. The entries along with their answers are arranged in the following table: 


\begin{tabular}{|l|l|}
\hline Metaphorical riddle & \\
\hline The riddle as the precedent & The explicit input space \\
\hline The answer as the sequent & The hidden sequent space \\
\hline $\begin{array}{l}\text { Shared properties between } \\
\text { the precedent and the sequent }\end{array}$ & The generic space \\
\hline $\begin{array}{l}\text { Metaphorical relation between } \\
\text { the precedent and the sequent }\end{array}$ & The blend space \\
\hline
\end{tabular}

After inserting different parts of the metaphorical riddles in the above table, we tried to illustrate different styles of metaphorical integrations in the riddles. It should be noted that the primary concern of this paper is to uncover the underlying conceptual structure of the Kurdish riddles; accordingly, we do not delve into analyzing their linguistic properties.

\section{TYPOLOGY OF KURDISH RIDDLES}

By analyzing the data according to the abovementioned methodology, it became evident that there are at least three different kinds of riddles, two of which can be categorized as metaphorical riddles with different conceptual structures, and the third one may be classified as non-metaphorical riddles. Let us take a look at the following cases characterizing these three groups.

7. Tâli hawreŝm, har lerawa tâ baghdâ aykeŝm.

A silk thread, I pull it from here to Baghdad. (road)

8. Gâyakm haya gâ bâri, nagoŝti daxore na bāri.

I have a cow that has a great load on its back; its meat and its load can't be eaten. (scarab beetle and its dung ball)

9. Hana no sini, ba har sini no nân, la sar har nân no kəfta, la sar har kəft no korpa.

I have nine trays, and nine loaves of bread on each tray, and nine kəftas (a kind of food) on each loaf of bread, and for each $k \partial f t a$, there are nine kinds to feed. (81 loaves of bread, 799 kəftas, and 6561 children)

Regarding riddle 7 as a member of the first sub-group of the metaphorical riddles, the phrase long silk thread has been used metaphorically, because the contextual meaning of the silk thread in this riddle (road) is different from its basic meaning ( $a$ kind of thread); besides, the former contrasts the latter, and the road is understood in comparison to the silk thread (for MIP see Praggle- 
jaz Group 2007). Then, it is concluded that this riddle is a metaphorical one. Consequently, it can be argued that the riddle includes the input space of (silk thread) as the precedent and that of (road) as the sequent. In the blend space, the image of the long silk thread and the image of the long road are compressed and combined with one another. The generic space of this conceptual integration involves shared properties like a geometrical shape of a long line. The sequent space of road is not explicit in the riddle, but the precedent space of long silk thread and its shared properties with the image of the road are presented in the riddle. The riddle provides the information for the riddlee to guess the road in a metaphorical way.

Considering riddle 8 as a member of the second sub-group of the metaphorical riddles, the word cow is used metaphorically. The contextual meaning of cow (which is scarab beetle) contrasts its basic meaning (a mammalian animal). Also, the former (scarab) as the contextual meaning is understood in comparison to the latter (cow) as the basic meaning. Accordingly, this riddle is a metaphorical one. The word load may not be regarded as a kind of metaphorical word because the load carried by the cow is not in contrast to the dung ball as a load carried by the scarab. The dung ball itself is a kind of load, so it is the hyponym of the load. Therefore, this riddle is a metaphorical one, simply because of the metaphorical relationship between cow and scarab.

The precedent including the cow and its load is mapped onto the sequent involving the scarab and its dung ball, and then they are combined and compressed into the blend space. The generic space contains the carrier and an object (the carried thing). The metaphorical integration between the precedent and the sequent is stated in the first part of the riddle in which the scarab beetle is described as a cow carrying a load. However, the second part of the riddle causes a disanalogy between the spaces. It says that the meat of this cow (scarab) cannot be eaten. According to Islamic rules, eating the meat of the cow is legitimate, but eating the scarab beetle is forbidden. Accordingly, it states that although the scarab beetle resembles the cow in some respects, the former is not edible. The second part of the riddle aims at highlighting the differences between the cow and the scarab beetle. Then, this riddle simultaneously makes a metaphorical analogy between the precedent and the sequent, and then highlights a disnalogy between them. How does this disanalogy occur along with the metaphorical integration between the precedent and the sequent? How can this complicated metaphorical structure be explained in terms of cognitive terminologies? In the chapter about discordant metaphors we tackle this problem in detail.

Example 9 as a member of the non-metaphorical riddles (the third group) includes a kind of mathematical computation. The words are not used meta- 
phorically in this example because the contextual and the basic meanings of the words are the same. Furthermore, the sequent is not understood in comparison to the precedent. In contrast, the sequent can be found via a mathematical computation of the precedent. All the other data are classified into these three groups.

Accordingly, it can be argued that the riddles existing in the book may fall into three different groups. The first group includes the riddles specified by a metaphorical relationship between the precedent and the sequent according to a kind of similarity between them. However, the second group includes those riddles containing a metaphorical relationship between the spaces along with a disanalogy between them. They consist of two contradictory parts: the first one shows the metaphorical relation between the precedent and the sequent, and the second one demonstrates a kind of contradiction between them. The only distinctive feature distinguishing the first and the second group of metaphorical riddles refers back to the disanalogy made in the second part of the riddle. The third group involves those riddles representing the answer in a non-metaphorical manner. The first group (illustrated by example 1) includes 37 riddles; the second group (see example 2) contains 30 riddles, and the third group (non-metaphorical riddles) involves 33 riddles. So, the first group constitutes about 37 percent, the second one about 30 percent, and the third one about 33 percent of all the riddles. In the following sections, we try to scrutinize the conceptual metaphorical structures of these groups in detail.

\section{The first group: Contingent metaphors}

The first group comprises 37 percent of the total number of riddles. It contains those riddles that are constructed by a metaphorical relationship between the precedent and the sequent. Consequently, the precedent space is mapped onto the sequent space. Then, they are mixed and compressed with one another in the blend space. The sequent space is not explicit in the linguistic structure of the riddle; therefore, the riddlee should guess the hidden sequent space mainly in terms of the conceptual and image-schematic properties of the precedent space. There are some other examples as follows:

10. Âsmâni nazmoka, ley dabare bafroka.

A near (low) sky from which snow is falling. (the sieve (and the flour falling from it))

11. Ble am ŝera hâsâna, mriŝkî̂ hali dene.

Say this easy poem that can be recited by a hen. (egg) 
12. Kotakayakyân haya ba du mât.

Two houses have just one column. (two nostrils and the septal nasal cartilage between them)

13. Tawilayakəm haya, diwâri čarma, siwdu mař teydâ, beharâw gərma. I have a stable, with a wall of leather, thirty-two sheep are there quietly. (mouth, cheek, and teeth)

14. Kar mardu, bâr zandu.

The donkey is dead, and the load is alive. (cradle and a baby)

15. Du mâri řâs, du črây gaŝ, du kona rewi, âw ley roi.

Two black snakes, two bright lamps, two fox dens through which water is passing. (eyebrow, eyes, nostrils, and nasal mucus)

16. Bâni hasâna, zeri qorâna.

Its upper surface is grindstone and its lower surface is Quran (a book). (mushroom)

17. Du âw la Jâme.

Two kinds of liquid in a cup. (egg with its white and yellow liquids)

18. Wak goy zawi wâya, wargi pər̆ la hawâya, hatâ tey hal day, aw la samâa.

It is like an earth globe, its belly full of air, if you kick (its ass) it will dance. (ball)

19. Ŝâreki ŝina, čwâr dawri sawza, xalki rangarang, newakay hawza. There is a green city that is surrounded by green walls, the people are in different colors, and there is a pool at the center. (water melon)

In riddle 10, the words are applied metaphorically. For example, the contextual meaning of the sky (sieve) contrasts its basic meaning (the space above the earth), and the former is understood in comparison with the latter. Just in the same way, the basic meaning of the snow (white pieces of the frozen water) is turned into the contextual meaning of the flour; and the flour is understood in comparison with the snow. This metaphorical riddle is shaped by a metaphorical mapping between the input spaces of sky and sieve. The precedent space includes mental images of the sky, white snowflakes, and also a dynamic image of snowing; and the sequent space involves a sieve, white particles of flour, and the falling of flour particles from the sieve. The elements of the input spaces are cross-mapped respectively, and then they are combined and compressed into the blend space. The generic space includes the shared elements such as a container, white matter, and a descending movement. This metaphorical riddle is based on such a conceptual metaphorical integration. 
The riddlee should find the right answer by examining different possible candidates (for the sequent space) that can integrate metaphorically with the precedent space. There are many possible candidates that bear similarities to the structure of the precedent space, and can participate in a metaphorical integration with it; therefore, the riddlee can imagine several possible answers for this riddle. For example, the snowflakes can be mapped onto salt particles, plaster particles, or onto other white materials which can be sifted. Also, the near sky can be mapped onto the salt shaker and other kind of sieves. Consequently, this riddle is specified by potentiality and indeterminacy of the sequent space, and may have more than one answer. But the riddlee wins, when he or she guesses the intended sequent space in the riddler's mind. As Ben-Amos puts it (1976: 249), it is the will of the riddler that determines the right answer of a riddle having many different potential answers (sequents). Then, there are many possible sequent spaces that are conditioned by the image-conceptual structure of the precedent space. Because of this contingency, the riddlee has to guess several times. No one loses the game by saying the false answer. They can guess several times until they find the right choice.

As for example 11, the words of poem and reciting the poem are used metaphorically. The contextual meanings of the poem and reciting the poem refer to egg and egg laying, and the contextual meanings contrast their basic meanings; and also the former is understood in comparison to the latter. A kind of metaphorical integration is made between the precedent space including the elements of a poet, recitation, and its product (a poem) and the sequent space involving a hen, egg laying and its product (the egg). The elements of the input spaces are cross-mapped, and then compressed and combined in the blend space. The generic space includes the shared elements like the producer, production, and the product. It is worth mentioning that one of the elements of the sequent space, namely hen, is evident in the riddle, but the hidden answer (egg) is not explicit.

The riddlee tries to guess the sequent by considering the precedent (poem and poet) and also by regarding one of the conceptual correspondences (hen as a poet reciting a poem). The riddlee should find an appropriate candidate suitable for mapping and blending with the precedent. Yet, there are some possible candidates other than the one determined by the riddler. For example, cluck is a good candidate for the poem of a hen, although it is not chosen by the riddler as the correct answer. The meat of a hen can be imagined as another candidate. After examining these possible sequents, the riddlee should choose the egg as the intended answer.

In riddle 12, the words column and house are applied metaphorically. Their contextual meanings (the septal nasal cartilage and nostril) contrast their basic 
meanings (a long solid stone and a building); and also, the former is understood in comparison to the latter. The precedent space involves the image of two houses having a same column, and the sequent space contains the image of two nostrils plus their septal nasal cartilage. The input spaces are crossmapped, and then combined and compressed into the blend space. Accordingly, a fusional metaphorical image emerges in the blend space. The generic space involves a kind of bipartite container. The riddlee should find out the intended sequent according to the clues supplied in the riddle. Besides the intended answer, several potential candidates can be imagined as the sequent space by the riddlee. For example, there is a traditional wooden cabinet in Kurdistan that has two rooms which are separated by a wooden column. It is another image which can be mapped onto the precedent space.

The words in riddle 13 are used as metaphorical words. The basic meaning of the stable refers to a kind of room in a building, but its contextual meaning in this riddle refers to the mouth. Although the basic meaning of the wall is a kind of structure, it refers to the cheek in this riddle. Finally, the contextual meaning of sheep is the teeth, while its basic meaning refers to a farm animal. All the contextual meanings of these three words are understood in comparison to their basic meanings. Accordingly, this riddle is based on three related metaphors in which the precedents of the stable, the wall, and the sheep are cross-mapped with the sequents of the mouth, cheek, and teeth respectively; consequently, they are mixed and compressed into their blend spaces. The generic space for the input space of the stable and the mouth includes an abstract container. Also, the generic spaces for the input spaces of the wall and cheek and the sheep and teeth include a flat structure covering a space, and some white round entities respectively. The large number of metaphors and clues in this riddle highly restricts the range of possible candidates for the sequents. The presence of another hint (with a wall of leather) intensifies this restriction. It implicitly describes the wall (cheek) as something made out of meat.

In riddle 14, the words donkey and load are used metaphorically because their contextual meanings (cradle and baby) contrast their basic meanings (an animal and a thing), and also, the former are understood in comparison to the latter. The precedent space includes the elements of a dead donkey and its load, and the sequent space contains the cradle and the baby. The generic space involves the abstract concepts of the carrier and the carried thing. The input spaces are cross-mapped, and then combined and compressed in the blend space. As the cradle is an inanimate entity, and the baby is animate, the riddle says the donkey is dead and the load is alive. The linguistic structure of the riddle includes the precedent space (donkey and load) and some of the metaphorical correspondences (the deadness of the cradle has been projected 
onto the donkey, and the animacy of the baby is projected onto the load). The riddlee should guess the predetermined sequent in terms of the precedent space and the explicit correspondences. Besides the cradle and the baby, several other potential sequent spaces can be imagined that may be mapped onto the precedent space. For example, a car and its driver, a bicycle and a rider, and even a bed and a human can be possible candidates for the sequent space.

The other examples in a similar way include a kind of image-conceptual integration, in which the input spaces of the precedent and the sequent are cross-mapped, and then are compressed in the blend space. In most of the cases, the riddlee can imagine many possible candidates for the sequent space, because many different candidates can take part in the conceptual integration with the precedent. So the process of guessing is not directed by a kind of logical necessity, but by a kind of potentiality and possibility, conditioned by the conceptual and image structure of the precedent space. The riddlee should examine many spaces as the possible candidates for the sequent space to guess the answer. Regarding the numerous candidates which may bear resemblance to the precedent, and can participate in a metaphorical integration with the precedent, the riddlee may guess more than one answer. It is worth taking into consideration that this possibility is conditioned by the general structure of the precedent. Due to this conditioned possibility, we call them contingent metaphors.

The parallelism between conceptual and image structures of the precedent space and the sequent one is the basis of this metaphorical integration. Considering the distinction between resemblance metaphors and correlation-based metaphors (Grady 1999), it can be argued that these metaphorical riddles are categorized as resemblance metaphors, because they are not motivated by any kind of experiential correlation between the spaces. They are based on a perceived similarity between the precedent and sequent spaces by the riddler. This resemblance is completely construed by the subjective perceptual judge of the riddler, and is not a pre-determined fact. The following diagram illustrates the conceptual integration of the riddles with contingent metaphors.

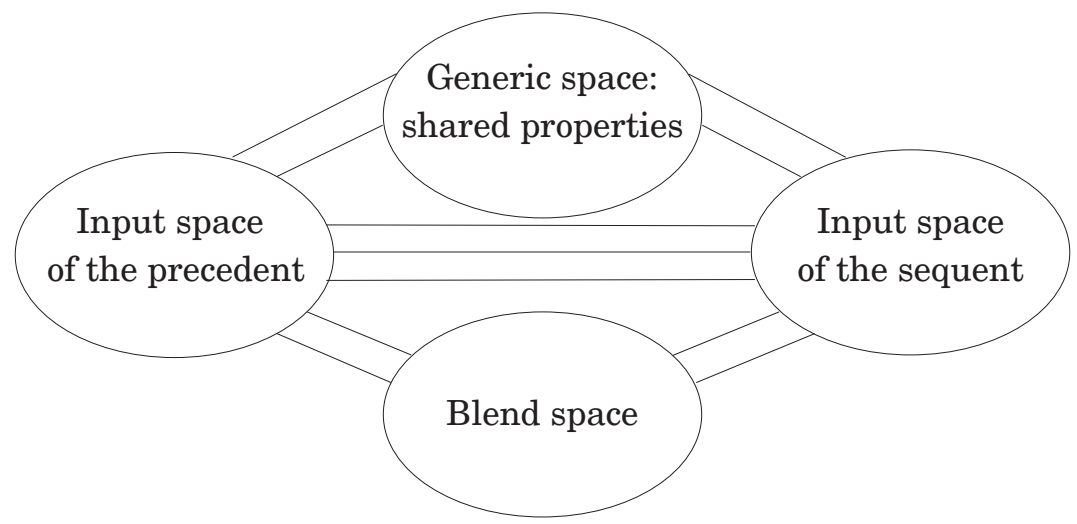


The linguistic manifestations of these riddles include the precedent space and some of the conceptual correspondences as clues for guessing the sequent space. Yet, the riddle does not refer explicitly to the hidden sequent space. When the riddlee mediates this kind of a riddle, he or she first considers it as a deliberate metaphor. As regards a deliberate metaphor, the speaker consciously "invites ... the addressee to ... set up a cross-domain mapping ... in order to view one thing in terms of something else" (Steen 2011: 84). The speaker invites the addressee to view the sequent space from the perspective of the precedent space. Accordingly, metaphorical riddles as deliberate metaphors invite the riddlee to guess the hidden sequent in terms of the conceptual and image-schematic properties of the precedent space. The deliberateness of the riddle leads the riddlee to make a metaphorical mapping between the precedent and any possible candidates for the sequent space.

\section{The second group: Discordant metaphors}

The second group comprises 30 percent of the total number of riddles. As mentioned, it includes two contradictory parts: the first part presents a metaphorical analogy between the precedent and sequent spaces, and the second one highlights a kind of disanalogy between them. Because of this contradiction, they are called contradictory riddles in the traditional classification (see Ahmadi 2010). These riddles use contradictory expressions for pointing out the intended answer. There are some other examples as follows:

20. Ârdi hâryâw, $\hat{a} \hat{s}$ naditu.

It is the milled flour, but has not been milled in a water mill. (snow)

21. Aw bar dâwew âws nya.

She delivers a baby, but she is not pregnant. (ear and earwax)

22. Mângâyaki bekaĺu gwân, sîrân dada ba hamuwâ.

It is a cow producing milk for everyone, but it has no udder. (bee)

23. Bahri du rang, na ŝapoli haya na dang.

A sea with two colors, but without any noises and waves. (egg)

24. Krâsi xayât naditu, ârdi âsyâw naditu, dâri najjâr naditu.

A cloth (fabric) not sewn by a tailor, flour not milled by a water mill, wood not worked out by a carpenter. (oleaster)

25. Zəmāni nya, qasān dakā.

It has no tongue, but it can speak. (pen) 
26. Na gaday haya na pâze, boy dačeta sar bane.

It has no belly and feet, but can go up to the roof. (smoke when it goes up)

27. Korekmân habu bedastu pâ.

We had a boy, he had no hands and feet. (egg)

28. Bolboleki xoŝâwâza, balam palawar nya.

It is a nightingale with nice songs, but it is not a bird. (târ, a musical instrument)

29. Klâwek be taqalu derumân.

It is a hat that is not sewn. (the eggshell on the head of a chicken)

The words are used metaphorically in riddle 20 , simply because the contextual meaning of the milled flour (snow) contrasts its basic meaning (powder of wheat), and also the snow as the contextual meaning is understood in comparison to the flour. The riddle includes two parts: the first describes the snow as a kind of milled flour; and the second says that this specific entity is not milled in the water mill. In fact, the riddle wants to visualize the sequent via contradictory phrases. First, let us consider the opening part of the riddle, which embodies the metaphorical integration between the precedent and the sequent. The riddle in the first section is based on a metaphorical integration between the precedent space including particles of flour and the sequent space involving snowflakes. These two input spaces are cross-mapped, with the result that snow corresponds to flour, the whiteness of snow to the whiteness of flour, and the fineness of snow to the fineness of flour. These relations and elements are combined and compressed into the blend space. The generic space includes the shared properties like tiny and white entities. This metaphorical integration is stated in the first part of the riddle which describes snow as milled flour. Now let us scrutinize the second part. The riddle in the second part makes a disanalogy between flour and snow. It says that this kind of milled flour (snow) has not been milled in a water mill. It means that, whereas the tiny particles of snow closely resemble the particles of the milled flour, the former is not milled by using a water mill. The second part highlights an important difference between the milled flour and the snowflakes. The first part refers to the metaphorical analogy between the precedent and the sequent, and the second one highlights a kind of contrast and disanalogy between them. The question is how this disanalogy becomes possible in spite of the pre-established metaphorical analogy. The disnalogy between the input spaces becomes visible by the process of backward projection from the blend space to the input spaces. The backward projection brings about disanalogy between spaces; it disrupts the compression and presents a powerful contrast between the spaces (see 
Fauconnier \& Turner 2002: 49, 308). Therefore, the disanalogy in this riddle emphasizes the difference between snow and flour, and makes a kind of conceptual distance among the pre-combined elements in the blend space. Via this disanalogy, the second part can show the differences between snow and flour by highlighting an important property of the second input space (being milled in a water mill) that is excluded from the blend space. Although, the procedure of integration brings the spaces together in a metaphorical way, the backward projection makes conceptual distance among them by highlighting a radical difference. Accordingly, the second part of the riddle manifests the decompression and disanalogy made by the procedure of the backward projection. The following diagram shows this complicated system of integration.

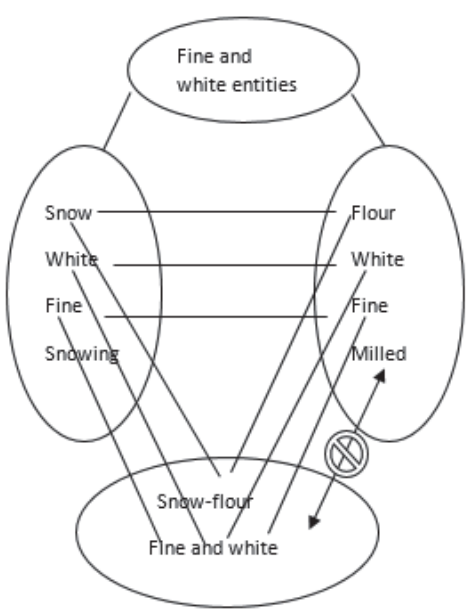

The arrow with the stop symbol directed from the blend space to the second input space depicts the process of a backward projection. It illustrates the process of highlighting the excluded property that seems like a surplus to the tight integrated system.

This diagram illuminates why this riddle cannot be explained by the conceptual metaphor theory (CMT), for this conceptual integration network is more than establishing a metaphorical integration between snow and flour. Besides the metaphorical integration, it provides disanalogy and decompression between the spaces by a backward projection. Because of these two simultaneous discordant processes, it seems that this metaphor denies some parts of itself. The linguistic structure of this riddle includes two parts that indicate the metaphorical integration and the disanalogy made by the backward projection. Although snow is not stated linguistically, it is integrated metaphorically with the flour in the first part of the riddle, and is implicitly contrasted with the milled flour 
in the second part. So the riddlee may use these two clues (metaphorical analogy in the first part and the disanalogy in the second part) to guess the hidden sequent space of snow. He or she tries to find a concept as an apt candidate for the sequent space which can take part in a metaphorical integration with flour, and also participate in a disanalogical relationship with the precedent.

The words in riddle 21 have been applied metaphorically, mainly because the contextual meanings of the woman and delivering a baby (ear and delivering the earwax) are in contrast to their basic meanings (a female human and bearing a baby), and, furthermore, the former are understood in comparison to the latter. This riddle includes two input spaces - pregnant woman as the precedent space and ear as the sequent space. Moreover, the potentiality of a woman to deliver a baby is compared to the potentiality of the ear to deliver earwax. The generic space includes the abstract concepts of the producer and the product. Then, the elements of these two input spaces are combined and compressed in the blend spaces. Consequently, a kind of metaphorical fusion is brought about. This is the result of the metaphorical integration between the input spaces. But in the second part, the riddle says that this entity delivers a baby, but it is not pregnant. In other words, it says that although as a pregnant woman it delivers a baby, it is not pregnant. Accordingly, some aspects of the metaphorical integration network are decompressed. This disanalogy between spaces is brought about by a backward projection from the blend to the inputs. It highlights the radical difference between the ear and the pregnant woman via highlighting the non-pregnancy of the ear in the sequent space. The linguistic structure of the riddle involves two parts: the first one indicates the metaphorical integration, and the second one illustrates the disanalogy. The concept of ear is not announced linguistically, but it is integrated with a pregnant woman in the first part, and implicitly contrasted with it in the second part. The riddlee should guess a candidate for the hidden sequent space that can take part in both of these processes.

In riddle 22 the words are used metaphorically. The contextual meaning of cow (bee) is in contrast to its basic meanings (a mammalian animal). Also, the former is understood in comparison to the latter. The riddle includes a precedent space including a cow and its milk and a sequent space involving the bee and its honey. The generic space includes the producer and its product. The precedent and sequent spaces are combined and compressed in the blend space. However, a kind of disanalogy between them is made by a backward projection. Accordingly, it says that this specific cow (bee) has no udder. The first part of the riddle contains a metaphorical integration, and the second one involves a disanalogy between the cow and the bee. The hidden sequent space should be guessed by considering these two parts. 
In riddle 23 the words are applied metaphorically. The contextual meaning of sea (egg) is in contrast to its basic meaning (a large area of water), and it is also understood in relation to the basic meaning. This riddle includes two input spaces of the sea as the precedent and the egg as the sequent. The generic space includes the abstract concepts of a container and liquid. The sea liquid is mapped onto the egg liquid, and then both are combined and compressed into the blend. Consequently, the egg as the hidden sequent space is described as a kind of sea in two colors (yellow and white). However, when the metaphorical integration is completed, a kind of disanalogy between the spaces is made by a backward projection. Accordingly, it says that while the egg liquid is described as the sea, it has no waves or sough. In other words, it wants to make a contrast between the sea liquid and the egg liquid. Then, it highlights the calmness and immobility of the liquid of the egg in contrast to the wavy and restless water of the sea. The riddlee should guess the sequent space by considering these contradictory descriptions made by a metaphorical integration and backward projection.

Riddle 24 includes metaphorical words too as the contextual meanings of the words (skin of oleaster for the cloth, seed for wood, dry flesh of oleaster for flour) contrast with their basic meanings, and also the former is comprehended in comparison with the latter. This riddle includes more than one integration network. The riddler wants to specify oleaster by different metaphors. First, he or she makes metaphorical integrations between the skin of oleaster and a cloth, between its seed and wood, and then between dry flesh of oleaster and flour. But there are also some disanalogies between all these integrated spaces by some backward projections. Via backward projections, it makes disanalogies between the cloth sewn by a tailor and the skin of oleaster not sewn by anyone, between the milled flour and the dry flesh of oleaster (which resembles the flour, but is not milled), and between the wood worked out by a carpenter and the wood-like seed of the oleaster not worked out by any carpenter. The riddlee tries to guess oleaster by considering all these metaphors. The riddle includes three metaphorical integrations and three backward projections. All these clues will help the riddlee to guess one possible sequent space in which its parts can participate in all of these three conceptual integrations.

Other examples of this group follow this conceptual procedure too. The riddles in this group include two parts of the precedent and sequent, which can be considered as two input spaces. Elements and relations of the input spaces are cross-mapped, and then they are combined and compressed in the blend space. As a result, a metaphorical integration between two different input spaces (precedent and sequent) takes shape, but then this riddle establishes a kind of disanalogy between the spaces. This is done by a backward projection, which 
disrupts the integration and compression of the spaces in the blend spaces. The backward projection is the process that induces a disanalogy and a powerful contrast between the input spaces. So this kind of a riddle first makes a metaphorical integration between the precedent and the sequent, and then makes a disanalogy between them by using a backward projection. We call these riddles discordant metaphors, because they simultaneously prove and deny the metaphorical integration. The following diagram illustrates the conceptual integration of the riddles with discordant metaphors. Symbol X in the diagram refers to the distinctive element highlighted by the backward projection.

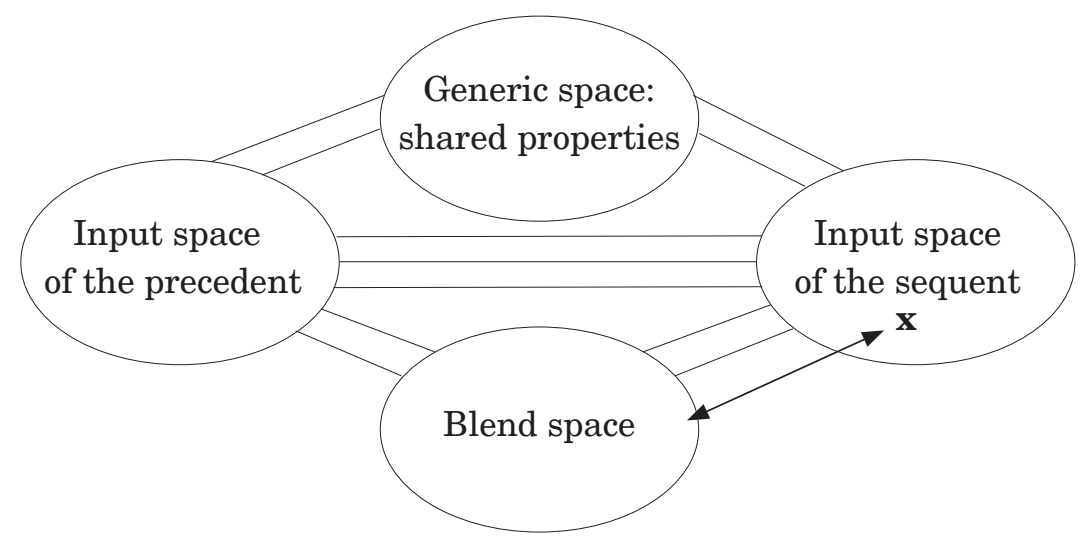

The linguistic structure of these riddles includes two parts; one indicates the metaphorical integration between spaces, and the other states the disanalogy between them made by backward projection. It describes the hidden sequent space as something similar and different in relationship to the precedent space. These concurrent contradictory descriptions bring about the contradictory discourse of these riddles. The linguistic manifestations of these riddles include the precedent space, but do not refer linguistically to the sequent space. The discordant metaphorical riddle as a deliberate metaphor invites the riddlee to guess the hidden space (sequent) in terms of the precedent space via an analogy brought about by metaphorical integration and a disanalogy made by backward projection. The riddlee should guess the hidden space (sequent) by examining those possible spaces that can take part in a metaphorical integration with the precedent space. Although there can be some potential candidates for the sequent space in the riddles, the simultaneous concurrent process (analogy done via metaphorical integration and disanalogy via backward projection) reduces the range of possible candidates to a considerable extent. 


\section{The third group: Non-metaphorical riddles}

The third group comprises 33 percent of the total number of the riddles. The riddles in this group do not use the metaphorical relationship between the precedent and the sequent in their structures. The most common topics of these riddles are mathematical computations, and guessing an object by considering some of its properties described in the riddle. Some riddles, by choosing a non-central member of a category, and by describing its non-prototypical and sometimes pseudo-contradictory properties, ask the riddlee to guess the name of that object. Because they are not shaped metaphorically, we do not delve into them. We hereby mention ten of them:

30. Hana no sini, ba har sini nine nân, la sar har nân no kəfta, la sar har kəft no korpa.

I have nine trays, and nine loaves of bread on each tray, and nine kəftas (a kind of food) on each loaf of bread, and for each $k$ fta, there are nine children to feed. (81 loaves of bread, 799 kəftas, and 6561 children)

31. Se ow penj, pazdaw du, hafdaw sew bist: dakâta čand.

Three and five, fifteen and two, seventeen and three: what is the result? (twenty)

Riddle 31 is based on a tricky computation. The riddle includes a series of figures, and operations like multiplying and adding. The riddler has not mentioned the mathematical operations. This riddle with its mathematical operations looks like: 3 multiplied by 5 equals 15, and 15 plus 2 equals 17, and then 17 plus 3 equals 20 . The riddler has already done the computation without mentioning the operations. The riddlee should discover the implicit mathematical operations in the riddle, namely, he or she should read this riddle by drawing on the mathematical frame.

32. Jâreki la bən âw dačma sar âw.

I suddenly go from the beneath to the surface of the water. (swimming)

33. La qawzay sawztra, la ŝakry ŝirin tra, la kârabây zardtra.

It is greener than sea wood, it is sweeter than sugar, and it is yellower than hay. (apricot)

This riddle describes some properties of the apricot in the different levels of its development until it is ripe. Apricot is green before ripening, and yellow when it is completely ripe; a ripe apricot may be sweeter than sugar.

34. La new malânda kâmyân ba sirira.

Which bird lactates? (bat) 
Bat is a non-central member of the bird category. It is a bird, but produces milk like mammals.

35. Penj farzay be bismilla.

A worship without bismillâ that is done five times a day. (azân)

Muslim's worships begin with bismillâ (in the name of god), but azân is the only worship that is not begun by bismillâ.

36. Məndâli haya bal̂̂m məndâli kas nabua.

He has many children, but he is not anyone's child. (Adam)

This riddle refers to the Adam figure in Islam.

37. Bâli nya, helka dakâ.

It has no wings, but it lays eggs. (snake)

The riddlee can guess more than one answer for this riddle. Turtle, lizard, and some others can be good candidates for the answer of this riddle.

38. Helka daka, mriŝk nya.

It lays eggs, but it is not a hen. (turtle)

The riddlee can imagine more than one answer to this riddle too.

39. Ham bukm ham xasum.

I am a daughter-in-law and a mother-in-law.

She is a person whose son has got married, thus she is the mother-in-law to her son's wife, and because her mother-in-law is alive, she is daughter-in-law to her husband's mother. It should be noted that there are some riddles in this group that can have more than one correct answer.

\section{SOME IMPLICATIONS FOR METAPHORICAL GUESSING}

As mentioned above, metaphorical guessing is realized in metaphorical riddles; accordingly, knowledge of the conceptual structure of metaphorical riddles can lead us towards some helpful assumptions about the procedure of guessing in metaphorical riddles. As there are two different groups of metaphorical riddles with different conceptual structures, let us scrutinize the process of guessing in them separately.

As mentioned earlier, the riddles with contingent metaphors are based on metaphorical mapping between the precedent space and the sequent one; the spaces are cross-mapped, and then combined and compressed into the blend 
space. The metaphorical integration between the spaces is motivated by a kind of visual similarity between them. The linguistic manifestation of the riddle includes the precedent space and some of the conceptual correspondences, but it does not refer directly to the sequent space. The riddle as a deliberate metaphor invites the riddlee to guess the sequent in terms of the precedent according to some sort of visual similarity between them. As a result, the riddlee knows that the precedent refers semantically to something beyond itself (hidden sequent) with regard to a possible similarity. He or she knows that the contextual meanings of the used words in the riddle are metaphorically in contrast with their basic meaning.

To find the hidden sequent space, the riddlee should examine those possible candidates for the sequent that may satisfy two conditions; it should bear a resemblance to the precedent space, it may also take part in a metaphorical integration with the precedent. There is more than one candidate that can satisfy these conditions; therefore, several possible candidates for the sequent space can be imagined by the riddlee. Accordingly, the riddlee makes many metaphorical integrations between the precedent space and every possible candidate for the sequent space. By examining different metaphorical mappings between the precedent space and several possible candidates, the riddlee tries to single out the desired option in the riddler's mind. Each of the metaphorical integrations between the precedent and the candidates emerges as a different guess about the riddle. Determining the right candidate is completely dependent on the riddler's decision. Then, it may be argued that the metaphorical guessing is nothing more than deliberately making metaphorical relationship between the precedent space and the possible candidates for the sequent. Consequently, it can be concluded that guessing is itself a kind of deliberate process of making a metaphor. Guessing includes the numerous metaphorizations in order to discover the hidden sequent space in the riddler's mind.

As we pointed out earlier, the riddles with discordant metaphors include two input spaces (precedent and sequent) that are combined and compressed in the blend space, and then a kind of disanalogy between them is made by a backward projection. The linguistic structure of these riddles includes two parts; one indicates the metaphorical integration between the spaces, and the other one indicates the disanalogy between them. The linguistic manifestations of these riddles include the precedent space and some of the conceptual correspondences, but do not refer linguistically to the sequent space. The discordant metaphorical riddle as a deliberate metaphor invites the riddlee to guess the hidden sequent space in terms of the precedent space with regard to the similarity between them. Then, the riddlee knows that the contextual meanings (the sequent) of the precedent space should be interpreted (guessed) 
according to a kind of metaphorical comparison with the basic meanings of the precedent space (the precedent) with regard to a kind of similarity between them. In other words, he or she knows that the precedent refers to a hidden sequent as a result of a metaphorical relationship.

The riddlee tries to guess the hidden sequent space by examining those possible candidates which can take part in a metaphorical integration with the precedent space because of their similarity to the precedent. There would be many possible candidates for the sequent space, therefore the riddlee tries to reduce the number of possible candidates by taking into account the disanalogy made by backward projection. Although the disanalogy highly restricts the number of possible candidates for the hidden space, it is not impossible to imagine more than one answer (hidden space) for some of these riddles. Then, in this group guessing metaphorically is done via several metaphorical integrations between the precedent space and several possible candidates for the sequent as the hidden space. The candidacy of the many potential candidates is conditioned by the visual structure of the precedent space and also by the differences brought about by disanalogy. In this group, guessing is accomplished via a deliberate process of developing a metaphorical relationship between the precedent space and possible candidates for the sequent space.

All in all, it can be concluded that the metaphorical guessing in both groups follows some shared steps. In the first step, the riddlee should take the riddle as a deliberate metaphor; thanks to this presupposition, the riddlee in the second step should imagine all of the possible candidates bearing a kind of resemblance to the precedent space, and can take part in a metaphorical integration with it. The third step refers to making many metaphorical integrations among the precedent space and the possible candidates for the sequent space. Each metaphorical integration emerges as a different guess taken by the riddlee. The fourth step refers to continuing these numerous metaphorizations until the desired sequent in the riddler's mind has been singled out. Then, it may be argued that the metaphorical guessing refers to a deliberate process of making a metaphor between a fixed precedent space and the possible candidates for the sequent space.

However, the process of metaphorical guessing may be guided by some clues and ideas in the riddles. One element of the hidden input space may be verbalized in the linguistic manifestation of the riddle. For example, in example 12 (I have a stable, with a wall of leather, thirty-two sheep are there quietly: mouth, cheek, and teeth), the phrase of with a wall of leather includes an important element of the hidden sequent that may help the riddlee to find the right answer. Also, he or she may use the images presented in the riddle to find the suitable candidates that may be matched with them. But as was already mentioned, the 
clues are always contingent; in other words, they cannot visualize the answer completely. It is the riddlee who should fill out this murky and blurred image by mapping it with all the suitable candidates that have the potentiality to make a metaphorical mapping with the precedent.

Now let us illustrate this metaphorical process of guessing via analyzing a riddle with a contingent metaphor: (kotakayakyân haya ba du mât: two houses have just one column: two nostrils and the septal nasal cartilage between them). The riddlee first considers this riddle as a deliberate metaphor; in other words, he or she knows that the images of the house and the column as the precedent space refer metaphorically to different images as a sequent space according to a kind of visual similarity between the spaces. In other words, he or she knows that the unknown contextual meanings of the words of the house and the column (hidden sequent space) are understood in comparison to their basic meanings (precedent space) according to a kind of similarity between them. Then, the riddlee tries to speculate about different images bearing a kind of visual similarity to the precedent space, and can also participate in a metaphorical integration with it. For example, he or she can imagine some candidates like a cabinet with two parts divided by a wooden column, the nose divided by the septal nasal cartilage, or even as two parts of the lung separated by the trachea. The riddlee examines deliberately metaphorical integrations between the fixed images of the precedent space (two houses and a column) and the various images of the possible candidates (cabinet, nose, and lung) for the sequent space. Accordingly, three different metaphorical conceptual integrations come into existence that are manifested as different guesses taken by the riddlee. He or she develops metaphorical relationships among the precedent space and the possible candidates until finding the right option. So, answering the riddle is done via deliberately making metaphorical relationships among the precedent space and the possible candidates for the sequent.

\section{CONCLUSION}

This study indicated that a considerable quantity of riddles in Kurdish are metaphorically shaped. Their compositions are shaped via different metaphorical conceptual integrations. The riddles in the first group (contingent metaphors) include the precedent and sequent spaces as input spaces that are cross-mapped, and then combined and compressed in the blend space. This metaphorical riddles are not motivated by any kind of correlation-based experience, but they are motivated by resemblance. Considering their potentiality for having more than one correct answer, they are called contingent metaphors. The riddler asks 
the riddlee to guess the sequent by examining different mappings between the precedent space and many other different spaces as possible candidates for the sequent. The riddlee can imagine several possible candidates for the sequent space, but this possibility is restricted by the image-conceptual structure of the precedent space. The second group contains discordant metaphors that are shaped by a complicated metaphorical integration. Firstly, these riddles develop a metaphorical integration between two different input spaces (precedent and hidden sequent), and then make a disanalogy between spaces by a backward projection. One part of the linguistic structure of this riddle indicates the metaphorical integration, and the other one shows the disanalogy. The two processes of metaphorical integration and backward projection restrict the numerous possible answers to the riddle to a considerable extent. The sameness and the difference brought about by these processes provide two different clues for the riddlee to guess the right answer. The riddlee should guess the hidden input space (sequent) by taking into account the metaphorical integration and the disanalogy. Discordant metaphors can provide both similarities and differences simultaneously. The third group is not shaped by any metaphorical integration. These non-metaphorical riddles just show some properties of an object and ask the riddlee to guess the object. Considering the conceptual structures of the metaphorical riddle, it is argued that the metaphorical guessing related to the metaphorical riddles is specified by some cognitive procedures. First, the riddlee takes the riddle as a deliberate metaphor. Then, he or she should imagine the possible candidates for the sequent space capable of participating in a metaphorical integration with the precedent space according to a kind of similarity between them. After that, he or she develops many metaphorical relationships between the precedent space and the possible candidates for the hidden sequent space. The process of numerous metaphorizations continues until the intended option has been singled out in the riddler's mind. The metaphorical guessing is defined as a deliberate process of making a metaphor between a fixed precedent space and various possible candidates for the hidden sequent space.

\section{NOTE}

1 In modern Kurdish society, television and other forms of media have replaced the ancient forms of entertainment (riddle and storytelling) in the Kurdish families. 


\section{REFERENCES}

Ahmadi, Ebrahim 2010. Farhangi Matalu Moamma la Kurdi. [Dictionary of Puzzles and Riddles in Kurdish.] Sanandaj: Entesharat Elmi Kalej.

Ben-Amos, Dan 1976. Solutions to Riddles. The Journal of American Folklore, Vol. 89, No. 352, pp. 249-254. http://dx.doi.org/10.2307/539691.

Dienhart, John M. 1999. A Linguistic Look at Riddles. Journal of Pragmatics, Vol. 31, No. 1, pp. 95-125. https://doi.org/10.1016/S0378-2166(98)00056-3.

El-Sharif, Ahmad 2012. Metaphors We Believe by: Islamic Doctorine as Evoked by the Prophet Muhammad's Metaphors. Critical Discourse Studies, Vol. 9, No. 3, pp. 231-245. http://dx.doi.org/10.1080/17405904.2012.688209.

Fauconnier, Gilles \& Turner, Mark 2002. The Way We Think: Conceptual Blending and the Mind's Hidden Complexities. New York: Basic Books.

Gomola, Aleksander 2010. From GOD IS A FATHER to GOD IS A FRIEND: Conceptual Integration in Metaphors for God in Christian Discourse. In: E. Tabakowska \& M. Choinski \& L. Wiraszka (eds.) Cognitive Linguistics in Action: From Theory to Application and Back. Berlin \& New York: Walter de Gruyter, pp. 387-408.

Grady, Joseph 1999. A Typology of Motivation for Conceptual Metaphor: Correlation vs. Resemblance. In: R. W. Gibbs \& G. J. Steen (eds.) Metaphor in Cognitive Linguistics. Amsterdam \& Philadelphia: John Benjamins Publishing Company, pp. 79-100. https://doi.org/10.1075/cilt.175.06gra.

Green, Thomas A. \& Pepicello, W. J. 1984. The Riddle Process. The Journal of American Folklore, Vol. 97, No. 384, pp. 189-203. http://dx.doi.org/10.2307/540184.

Harries, Lyndon 1971. The Riddle in Africa. The Journal of American Folklore, Vol. 84, No. 334, pp. 377-393. http://dx.doi.org/10.2307/539632.

Harries, Lyndon 1976. Semantic Fit in Riddles. The Journal of American Folklore, Vol. 89, No. 353, pp. 319-325. http://dx.doi.org/10.2307/539444.

Khaleqi Mutlaq, Jalal 2002. Sukhanhaye Dirineh: Si Guftar dar Bareye Ferdowsi va Shahnameh. [Ancient Discourses: 30 Essays on Ferdowsi and Shahnameh.] Edited by Ali Dehbashi. Tehran: Afkar.

Köngäs-Maranda, Elli 1971. The Logic of Riddles. In: P. Maranda \& E. K. Maranda (eds.) Structural Analysis of Oral Tradition. Philadelphia: University of Pennsylvania Press, pp. 198-232.

Kovecses, Zoltán 2004 [2000]. Metaphor and Emotion: Language, Culture and Body in Human Feeling. Cambridge: Cambridge University Press.

Kovecses, Zoltán 2005. Metaphor in Culture: Universality and Variation. Oxford \& New York: Cambridge University Press.

Kovecses, Zoltán 2010 [2002]. Metaphor: A Practical Introduction. Oxford \& New York: Oxford University Press.

Lakoff, George 2007. The Contemporary Theory of Metaphor. In: V. Evans \& B. Bergen \& J. Zinken (eds.) The Cognitive Linguistics Reader. London \& Oakville: Equinox, pp. 267-315.

Lakoff, George \& Johnson, Mark 2003 [1980]. Metaphors We Live By. Chicago \& London: University of Chicago Press. 
Pragglejaz Group 2007. MIP: A Method for Identifying Metaphorically Used Words in Discourse. Metaphor and Symbol, Vol. 22, No. 1, pp. 1-39. http://dx.doi. org/10.1080/10926480709336752.

Rapone, Amanda 2012. Metaphorical Riddles as Conceptual Blends: A Look at the Riddle Solving Process. Language and Creative Mind. 11th Conceptual Structure, Discourse, and Language Conference. Vancouver, British Columbia, May 17-20, 2012. Available at http://csdl2012.sites.olt.ubc.ca/2012/04/16/metaphoricalriddles-as-conceptual-blends-a-look-at-the-riddle-solving-process/, last accessed on 12 September 2018.

Sarseyfi, Reza 2010. Pandi Peshiniani Kurdi: Masal, Matalu Benarati Masalay Kurdi. [The Wisdom of Ancient Kurdish People: A Dictionary of Kurdish Advice and Riddles.] Saqqhez: Gotar.

Shohani, Alireza 2014. Tabaqebandiye Chistane Amianeye Ilami. [The Classification of Ilami Riddles.] Majalleye Farhang va Adabyate Amme [Journal of Culture and Folk Literature], pp. 135-158.

Steen, Gerard 2011. From Three Dimensions to Five Steps: The Value of Deliberate Metaphor. Metaphorik.de, Vol. 21, pp. 83-110. Available at https://www.researchgate. net/publication/235325095/download, last accessed on 7 September 2018.

Weiner, E. Judith \& Palma, Paul de 1993. Some Pragmatic Features of Lexical Ambiguity and Simple Riddles. Language and Communication, Vol. 13, No. 3, pp. 183-193. http://dx.doi.org/10.1016/0271-5309(93)90025-I.

Zolfaqhari, Hasan 2013. Rikht Shenasiye Chistanhaye Manzume Mohalli. [Morphology of Local Poetic Riddles.] Majalleye Farhang va Adabyate Amme [Journal of Culture and Folk Literature], pp. 93-118. 